\title{
Testing Times: A symposium on the ethics and epistemology of animal experimentation
}

\author{
Sydney, Australia, September 20-21, 2011
}

\begin{abstract}
"Testing Times: A symposium on the ethics and epistemology of animal experimentation" (TT) was held over two days at Macquarie University, Sydney, Australia in late September. The meeting drew together a diverse group of participants including veterinarians (academics and practitioners), scientists, philosophers, members of animal ethics committees, and others to hear presentations on a range of issues related to animal experimentation and to discuss mechanisms to improve experimental practice.

TT was a "Minding Animals" (MA) Preconference Event with the next MA conference scheduled for July 4-6, 2012 in Utrecht, The Netherlands (www.mindinganimals.com). In keeping with the philosophy of MA, TT was successful in facilitating communication between traditionally disparate disciplines. Three main themes emerged from papers delivered at the meeting. These revolved around: i) examining fruitful links between research and experimentation on human and nonhuman animals; ii) new approaches to medical research and animal experimentation; and iii) the need for a more inclusive discussion of the ethics of animal experimentation.
\end{abstract}

\section{Links between human and nonhuman animals in research}

Hope Ferdowsian's paper asked participants to consider similarities across species in terms of empirical capacities, e.g., to feel pain, to suffer, and to have interests, and how nonhuman animals might be construed as a vulnerable population. In research on human subjects, the concept of vulnerability has prompted the development of a principle-based approach to experimental practice, but to date animal vulnerability has generated no similar response. Ferdowsian challenged us to redress this situation and construct a principled approach to animal experimentation by either applying principles developed in the human context to animals or by devising a new set of principles that apply exclusively to animals or to human and nonhuman animals alike.

Imke Tammen's presentation focused on similarities between human and nonhuman animals in terms of a particular disease. Neuronal Ceroid Lipofuscinosis (NCL) or Batten disease is common to humans as well as sheep, cattle, dogs, cats, horses, pigs, goats, ferrets, and mice. In her paper she posed the question "Are these animals 'good' models for human disease?" Tammen cautioned that this was not merely a scientific question pertaining only to the validity of models, but had an inherent moral dimension. Drawing on her own experience as a veterinarian and the ethical dilemmas her research generated, Tammen argued for the importance of researchers being educated in ethics. In her view, scientific practice should be informed by critical moral reflection.

Chris Degeling also explored the overlap between human and nonhuman animals through the lens of surgical research. He noted that despite the manner in which these domains had mutually informed each other, in general the professional histories of neither biomedical nor veterinary research detail how medically relevant knowledge is created through work in animals, or how it goes on to inform practice. He explained how models have a life of their own and can behave in unanticipated ways, some of which can only be appreciated in retrospect. Difficulties are therefore inherent in translation from models to targets, and the limitations of models need to be understood and appreciated by researchers.

\section{New approaches to medical research and animal experimentation}

A number of papers argued for a change in experimental practice with animals, for a diverse range of reasons. Ann Baldwin made a case for enriching the cage environment of rodents based on scientific grounds, but with attendant and favorable animal welfare implications. She described two studies - one with rats and another with gene knockout mice, both of which showed that cage enrichment changed the data obtained from these creatures. For instance diurnal rhythm, a phenomenon long associated with research in rodents, was shown to be merely an artifact of animal housing. Baldwin's work calls into question the reliability of results obtained from rodents in un-enriched environments.

Building in part on the work of Baldwin and others regarding the epistemological imitations of animal experimentation as routinely practiced, and in response to some of its ethical challenges, Jane Johnson's paper outlined a new model of animal experimentation - the animal-patient. On this model animals can be enrolled into experimentation in a manner akin to human participants in multicenter clinical research trials if they suffer from analogous diseases to humans (e.g., the animals Tammen uses in her work on NCL would qualify). It was argued that the use of animal patients would address many of the shortcomings in the quality of data produced in animal experimentation, as well as meet concerns about harms and possible injustices to animals in biomedical research. 
K-lynn Smith's work not only revealed hitherto unanticipated capacities in chickens, but offered a different model for experimental engagement with animals. Studies of fowl in captivity show they have greater cognitive skills than previously thought, including inferential and proto mathematical abilities, as well as a capacity to use signaling in strategic ways. Due to the divergent goals of behavioral and biomedical research, animals are treated quite differently in these spheres. The manner in which they are treated in the former could be instructive to researchers in the later field. In addition to adopting the 3Rs in practice, researchers in Smith's group add a $4^{\text {th }} \mathrm{R}$ - Retirement, and seek to re-house their fowl when their experimental work lives are complete.

Smith's work and that of other researchers on birds was featured in the film Thoughtful birds in action shown as part of the symposium. The work represented in this documentary includes tool use among New Caledonian crows and helps challenge the idea of nonhuman animals as vastly cognitively inferior to humans.

Brett Lidbury's proposal (in collaboration with Alice M. Richardson) was for rethinking medical research and its routine dependence on the rodent model. A particularly challenging area for animal replacement involves fundamental medical research, but Lidbury argued it was theoretically possible to remove nonhuman animals even in this research domain. He has adopted an absolute replacement approach, to both explore conceptually and to develop an alternative system for consideration by fundamental researchers. The paper showed how some rodent models might be replaced by using sophisticated in silico data mining and pattern recognition methods on human health data, in tandem with laboratory validation studies and analyses of genetic databases. The fundamental aims are the same as for animal-based medical research (to identify disease genes and pathways), but rely on in silico methods to deal with whole organism complexity, rather than inbred or genetically-modified mice.

Another paper which proposed a radical shift in the experimental status quo was presented by Matthew Chrulew (with Dominique Lestel and Jeffrey Bussolini). The paper was critical of the way institutionalized animal science is practiced with its focus on mechanisms and universality, reductionism and standardized ways of collecting evidence and assessing validity. Using insights from phenomenology, it was suggested that a bioconstructivist ethology might be developed, focused on the animal as subject engaged in inter-subjective interactions with a living observer.

Dinesh Wadiwel's paper drew on insights from Donna Haraway's work in an attempt to rethink animal experimentation and to overcome the prevailing stalemate between welfare and rights in this context. Wadiwel suggested Haraway's approach was fruitful in challenging the pervasive individualistic liberal ideal of freedom and the relationship between animals and nature, but that it had important limitations with respect to the conceptualization of violence towards animals.

\section{A more inclusive discussion of the ethics of animal} experimentation

Three of the papers presented advocated increased awareness of ethical issues surrounding animal experimentation and pro- posed mechanisms to enable a more inclusive discussion of the practice. In recognition of the complexity of decision-making in the area of veterinary research and animal use, Kate Millar's paper discussed using a structured approach to reflection and decision-making. Millar and colleagues have developed "The Ethical Matrix" - a framework and tool to help identify and explore the ethical dimension of contentious issues such as those around animal research. Interesting questions were also raised by Millar regarding the role of bioethicists in this context - should they be moral arbiters, teachers, or facilitators of ethical reflection?

In his paper John Hadley argued for the discussion of animal experimentation to be better informed and more transparent. Hadley grounded his view in the obligations of animal researchers involved in public communication about their work, as well as in the benefits to individual well-being and public policy of engagement with challenging issues like animal experimentation. His proposal involved making animal use data already available on the public record more easily accessible.

Denise Russell's paper also concerned the quality of ethical discussions involving animal research, this time in the context of Animal Ethics Committees (AECs). In her experience, AECs in Australia do not function to provide effective ethical scrutiny of scientific and medical research involving nonhuman animals. This is partly because they may come at the wrong time in the research process; there may be vested interests at stake; and there is no real opportunity to discuss the ethical questions raised nor to properly examine alternatives to the use of animals (e.g., funding has already been awarded to the project, therefore, AECs are under pressure not to block the project or to push for significant changes in methodology, etc.).

How to ensure a more inclusive discussion of the ethical issues in animal experimentation was also embraced in the final discussion session of the symposium with delegates making suggestions about which groups should be represented in the discussion (e.g., students, sociologists of science, science communicators, etc.), how they might be encouraged to participate and what techniques, tools and resources might facilitate discussion. Time was given over to thinking through the drivers for change in this area with economics noted as an important one, as well as researcher education. It was observed that having greater flexibility at the institutional level in terms of training and career paths is important so that researchers don't become locked into routinized use of animals, and are instead aware of (and capable of deploying) alternatives.

The symposium ended with agreement that further interdisciplinary forums and collaborations were needed to discuss issues arising in animal experimentation and to develop and improve the practice of animal research both ethically (especially in terms of how and if animals are used) and epistemologically, so results are applicable to human and nonhuman animals alike.

Jane Johnson and Brett A. Lidbury 